\title{
Introduction to statistical philosophy
}

Statistics

- We use statistics to confirm effects, estimate parameters, and predict outcomes

- The last 2 times I came to Cape Town, it rained, but only on Sunday

- Confirmation: In Cape Town, it rains more on Sundays than other days

- Estimation: I think it has rained on $>30 \%$ of Sundays in Cape Town

- Prediction: I don't think it will rain on Tuesday

- The last 20 times I came to Cape Town, it rained, but only on Sunday

\section{Confirmation}

- We want to know why prevalence increased in this village from $25 \%$ to $67 \%$.

- First ask: what are the data?

$-1 / 4$ to $4 / 6$

$-5 / 20$ to $12 / 18$

- $75 / 300$ to $180 / 270$

\section{Confirmation example}

- We measure the average heights of children raised with and without vitamin A supplements

- What sort of test could we do to confirm whether we believe this difference is due to chance? 


\section{Frequentist approach}

- Make a null model

- Test whether the effect you see could be due to chance

- What is the probability of seeing exactly a $1.52 \mathrm{~cm}$ difference in average heights?

- Test whether the effect you see or a larger effect could be due to chance

\section{Frequentist conclusions}

- If your effect size is unlikely to be caused by chance, you can believe the effect

- If your effect could easily be caused by chance, don't believe the effect

- But don't conclude that there is no effect

\section{Why don't we accept the null hypothesis?}

- Why do we reject the null hypothesis?

\section{Low $P$ values}

- What causes low P values?

- Large differences

- Lots of data

- Less noise

- A well-specified model

- Ability to disentangle covariates 


\section{High $\mathbf{P}$ values}

- What causes high $\mathrm{P}$ values?

- Small differences

- Less data

- More noise

- An inappropriate model

- Ability to disentangle covariates

\section{A high $P$ value is not evidence for anything!}

- Ever

- What kind of evidence should we use instead?

- Confidence intervals

- "Non-inferiority", "non-superiority", or both

* A low $\mathrm{P}$ value rejecting the idea that the difference is large

\section{Confidence intervals}

- How do we estimate the size of an effect

- Frequentists estimate confidence intervals by asking which values could be falsified (if they were considered as null hypotheses)

- Example:

- 12/30 women observed are HIV positive. What is my estimate for the prevalence?

- What if it's 120/300?

* What else do we need to know? 


\section{Frequentist philosophy}

- I am absolutely convinced that any two populations I can describe are different.

- I will never accept the null hypothesis! At least in biology

- Why then does it make sense for me to test the null hypothesis?

- One village has $12 / 25$ positive tests, another has 10/27. What should I conclude?

\section{Example: flipping a coin}

- I flip a coin 8 times, and get heads 8 times. Is the coin fair?

- A frequentist would do the same calculation if they were just handed the coin by a magician, or if they stole it while touring the mint

- A Bayesian needs a starting point to model from

- My a priori assumption is that there is a 1 in 800 probability that the coin has two heads (otherwise, it's fair)

- What do I think after flipping 8 heads?

\section{The Bayesian approach}

- A Bayesian approach to statistics requires modeling what you think is happening, not just a null model

- Much "bolder" than a frequentist approach

- We assume more, and we can conclude more, including predictions of the future 


\section{Bayesian inference}

- We want to go from a statistical model of how our data are generated, to a probability model of parameter values

- Requires prior distributions describing the assumed likelihood of parameters before these observations are made

- Use Bayes theorem to calculate posterior distribution - likelihood after taking data into account

\section{Bayesian Advantages}

- Assumptions more explicit

- Probability statements more straightforward

- Very flexible

- Can combine information from different sources

- Can make rigorous predictions about the future

\section{Bayesian Disadvantages}

- More assumptions required

- Lacks elegance of permutation approaches

- More difficult to calculate answers

\section{Prior distributions}

- You should usually start with a prior distribution that has little "information"

- Let the data do the work

- The "posterior" from one analysis can be the prior for the next analysis 


\section{$P$ values}

- Bayesian $\mathrm{P}$ values have a more direct interpretation than frequentist $\mathrm{P}$ values:

- We calculate the posterior probability that our effect size is positive

- If we are willing to rely on our assumptions, this gives the actual probability that our hypothesis is true

- We can also reject our hypothesis directly if the probability that it's true is smaller than a pre-specified value (although people usually don't do this)

\section{Credible intervals}

- Credible intervals are the Bayesian analogue of confidence intervals

- Since a Bayesian model is a complete probability model, the credible interval is simply an interval that we believe contains the correct answer with probability $95 \%$ (half of that probability is on each side of our median estimate).

\section{A concrete example}

- I observe 3 shooting stars in one hour of observing the sky.

- What is my credible interval for the rate of shooting stars?

\section{Shooting stars}

- For each rate, our likelihood of observing $N$ events in time $T$ if the true rate is $r$ is a Poission distribution with mean $r T$ :

$$
-\frac{(r T)^{N} \exp (-r T)}{N !}
$$

- We choose an improper, uniform prior over $\log r$, equivalent to $\pi(r)=$ $1 / r$.

- The posterior distribution is then proportional to:

$-(r T)^{N-1} \exp (-r T)$, which gives a gamma distribution with mean $N / T$ (the observed rate), and $\mathrm{CV} 1 / \sqrt{N}$. 


\section{MCMC sampling}

- Bayesian methods are very flexible: We can write down reasonable priors, and likelihoods, to cover a wide variety of assumptions and situations

- Unfortunately, we usually can't solve exactly

- Instead we use Markov chain Monte Carlo methods to sample randomly from the posterior distribution

- Simple in theory, but may be difficult in practice

- You may not even know whether you have calculated for long enough 